\title{
Acute kidney injury and mortality in coronavirus disease 2019: results from a cohort study of 1,280 patients
}

\author{
Natalia Chebotareva ${ }^{1}$, Svetlana Berns ${ }^{2}$, Angelina Berns $^{1}$, Tatyana Androsova $^{1}$, Marina Lebedeva ${ }^{1}$, \\ Sergey Moiseev ${ }^{1}$ \\ ${ }^{1}$ Tareev Clinic of Internal Diseases, Sechenov First Moscow State Medical University, Moscow, Russia \\ ${ }^{2}$ Moscow State Budgetary Healthcare Institution, Zhadkevich City Clinical Hospital, Moscow, Russia
}

Background: The development of acute kidney injury (AKI) in patients with coronavirus disease 2019 (COVID-19) is associated with a high risk of death. Published data demonstrate the possibility of severe kidney injury in patients suffering from COVID-19. However, these data are still controversial.

Methods: A total of 1,280 patients with a proven diagnosis of COVID-19 were included in our study. COVID-19 was confirmed in all patients using reverse transcriptase polymerase chain reaction test of a nasopharyngeal swab, and based on the typical computed tomography findings. Demographic data, underlying comorbidities, and laboratory blood tests were assessed. We assessed the incidence of AKI and its associated mortality defined by survival status at discharge.

Results: Proteinuria was identified with 648 patients (50.6\%) with COVID-19. AKI was identified in 371 patients (29.0\%). Ten of these patients (2.7\%) required dialysis. The risk factors for AKI included age of $>65$ years, augmentation of C-reactive protein, ferritin and an increase in values of activated partial thromboplastin time. Overall, 162 of the 1,280 hospitalized patients (12.7\%) and 111 of the 371 patients (29.9\%) with AKI did not survive. The hazard ratio (HR) for mortality was 3.96 (95\% confidence interval, 2.83-5.54) for patients with AKI vs. no AKI.

Conclusion: AKI was a relatively common finding among patients with COVID-19. The risk factors for AKI in COVID-19 included old age, the inflammatory response, the severity of lung involvement, and disseminated intravascular coagulation. These same factors, in addition to arterial hypertension, were found to increase the risk of mortality.

Keywords: Acute kidney injury, COVID-19, Hematuria, Mortality, Proteinuria

\section{Introduction}

The recently detected zoonotic coronavirus disease 2019
(COVID-19) is a novel virus that is closely related to severe acute respiratory syndrome coronavirus (SARS-CoV) and Middle East respiratory syndrome coronavirus (MERS-CoV).

Received: July 19, 2020; Revised: February 1, 2021; Accepted: February 2, 2021

Editor: Young-Ki Lee, Hallym University, Seoul, Republic of Korea

Correspondence: Natalia Chebotareva

Tareev Clinic of Internal Diseases, Sechenov First Moscow State Medical University, Rossolimo 11/5, 119435 Moscow, Russia. E-mail: natasha tcheb@mail.ru

ORCID: https://orcid.org/0000-0003-2128-8560

Copyright (C) 2021 by The Korean Society of Nephrology

(a) This is an Open Access article distributed under the terms of the Creative Commons Attribution Non-Commercial and No Derivatives License (http:// creativecommons.org/licenses/by-nc-nd/4.0/) which permits unrestricted non-commercial use, distribution of the material without any modifications, and reproduction in any medium, provided the original works properly cited. 
This coronavirus was initially detected in China in December 2019, after which it spread all over the world. COVID-19 is a severe acute respiratory syndrome that can involve alveolar and interstitial pneumonia. However, other organs may also be involved, including the kidneys, heart, gastrointestinal tract, and nervous system [1].

Acute kidney injury (AKI) is a serious complication of COVID-19, which also determines the prognosis of the disease. Various studies have suggested that the frequency of AKI with COVID-19 varies from $0.5 \%$ to $28 \%$ [1-3]. Wang et al. [4] observed no obvious renal function abnormality or AKI in more than 100 patients with COVID-19 during their admission to the hospital. However, despite their descriptions, many other studies have demonstrated that the development of AKI is associated with a high risk of death in patients with COVID-19 [1,3,5].

Previous coronavirus infections, such as SARS-CoV and MERS-CoV, have a high degree of sequence homology to SARS- CoV-2, and can also cause AKI that is associated with adverse outcomes. AKI, which affected $26.7 \%$ of MERS-CoV patients, was a risk factor for mortality [6]. In contrast, $17 \%$ of patients with SARS were diagnosed with AKI.

The development of AKI during the course of the SARS infection was associated with catastrophic outcomes, such as multiple organ failure (MOF) and death [7]. Multiple studies similarly have suggested that COVID-19 can be complicated by AKI; however, these data are still controversial. Therefore, there is a need for additional studies.

\section{Methods}

\section{Study population}

Between April and June 2020, COVID-19 patients who were admitted to the City Clinical Hospitals of Moscow Healthcare Department were selected for enrollment in this study. The study was approved by the local Ethics Committee of the Moscow State Budgetary Healthcare Institution (No. 0720 ) and the written informed consent from the patients was not required.

The included patients had a laboratory-settled SARSCoV-2 infection and viral pneumonia. The confirmed cases of COVID-19 were defined by a positive reverse transcriptase polymerase chain reaction (RT-PCR) test that was obtained by direct analysis of a nasopharyngeal swab. All of these patients also had typical images on computed tomography (CT) scan of the lungs. The typical radiographic features of coronavirus include a white mist or "ground glass" pattern in both lungs. In patients with a negative RT-PCR result, SARS$\mathrm{CoV}-2$ pneumonia was defined by severe acute respiratory infection with typical CT scan findings [8] and no other obvious etiology.

Demographic and clinical data, underlying comorbidities, and laboratory blood tests (including chemistry blood analysis, coagulation tests, assessment of renal function, C-reactive protein $[\mathrm{CRP}]$, lactate dehydrogenase $[\mathrm{LDH}]$, ferritin, and routine urine analysis) were collected. The international normalized ratio (INR), activated partial thromboplastin time (aPTT), ferritin, LDH, D-dimer, and CRP levels were evaluated at admission, and from baseline to maximum laboratory values.

Thoracic CT scan results and patient treatments (i.e., retroviral therapy, glucocorticoid administration, breathing and renal support) were obtained from electronic medical records. These data were transcribed into data compilation tables.

Proteinuria levels were measured in spot urine in g/g creatinine ( $\mathrm{Cr}$ ) by the colorimetric method using pyrogallol red (cutoff value, $0.15 \mathrm{~g} / \mathrm{g} \mathrm{Cr}$ ) Proteinuria $>0.15-1.0 \mathrm{~g} / \mathrm{g} \mathrm{Cr}$ was defined as mild, $>1.0-3.0 \mathrm{~g} / \mathrm{g} \mathrm{Cr}$ as moderate, more than 3.0 $\mathrm{g} / \mathrm{g} \mathrm{Cr}$ as severe. Changes in proteinuria levels were assessed in 279 patients during their hospitalization.

The dynamics of fibrinogen and platelet levels were evaluated in 906 patients during their hospital stay. Increasing D-dimer levels were associated with an increase in aPTT and a decrease in platelet count according to the criteria of the International Society on Thrombosis and Haemostasis. These changes were attributed to signs of disseminated intravascular coagulation (DIC). We identified and focused on preexisting comorbidities such as diabetes mellitus and systemic arterial hypertension, CT involvement score, and percentage of lung involvement.

\section{Outcome definitions}

The primary endpoint was AKI, which was defined according to the Kidney Disease: Improving Global Outcomes (KDIGO) Clinical Practice Guideline for AKI [9]. The standard definition of AKI in adults was designated as follows; an increase in serum creatinine (SCr) by $\geq 0.3 \mathrm{mg} / \mathrm{dL}$ within 48 hours, an 
increase in SCr to $>1.5$ times baseline within the previous 7 days, or urine volume $<0.5 \mathrm{~mL} / \mathrm{kg} / \mathrm{hr}$ for $>6$ hours. The severity of AKI was staged according to the following criteria: stage 1, SCr 1.5-1.9 times baseline or $\geq 0.3 \mathrm{mg} / \mathrm{dL}$ increase; stage 2, SCr 2.0-2.9 times baseline; stage 3, SCr 3.0 times baseline, an increase in SCr to $\geq 4.0 \mathrm{mg} / \mathrm{dL}$ or initiation of renal replacement therapy [9]. Recovery from AKI was identified by comparing the last creatinine level with the baseline creatinine level and was divided into stages 1,2 , or 3 .

According to the percentage of lung tissue involvement, the severity of pneumonia was determined as mild, moderate, severe, or extremely severe, as follows: mild involvement (CT1) was defined by involvement of up to $25 \%$ of the lung parenchyma; moderate (CT2) involvement involved $25 \%$ to $50 \%$ of the lung parenchyma; severe (CT3), $50 \%$ to $75 \%$; and extremely severe (CT4), more than $75 \%$ of the lung parenchyma. Acute respiratory distress syndrome (ARDS) was diagnosed in cases in which the $\mathrm{O}_{2}$ saturation decreased to $<92 \%$ and there was a need for respiratory support. Septic shock was diagnosed based on MOF and the need for vasopressor support.

We finally assessed mortality during hospitalization, which was defined by survival status on the day of discharge.

\section{Statistical analysis}

The results are presented as medians and interquartile ranges (IQR) for continuous variables with a non-normal distribution. Differences in the proportions and continuous data were tested using the Pearson chi-square test and Mann-Whitney U test, respectively. The cumulative incidence of AKI was estimated using Kaplan-Meier analysis.

The principal components analysis and rotation method varimax with Kaiser normalization were used to choose the number of components to extract. Linear regression models with the following covariates were used to estimate the risk factors for AKI; age, body mass index, changes in INR, aPTT, CRP, ferritin, fibrinogen, LDH levels over time (from baseline to maximum laboratory values), maximum D-dimer levels, maximum proteinuria, potassium levels, CT stage, presence or absence of diabetes mellitus, arterial hypertension, and ARDS.

The association of AKI and AKI severity with mortality over time was analyzed using uni- and multivariate Cox proportional hazards analysis with the following covariates; AKI, age, sex, body mass index, changes in INR, aPTT, CRP, ferritin, fibrinogen, D-dimer, LDH levels over time (from baseline to maximum laboratory values), maximum proteinuria, CT stage, presence and absence of SARS, diabetes mellitus, and arterial hypertension. HRs with $95 \%$ confidence intervals (CIs) for mortality were calculated. A p-value of $<0.05$ was considered statistically significant. The statistical analysis was performed using the IBM SPSS version 23 (IBM Corp., Armonk, NY, USA).

\section{Results}

\section{Demographic characteristics}

A total of 1,280 patients with a proven diagnosis of COVID-19 were included in our study. The average age of the hospitalized patients was 63 years (IQR, 52-75 years); 644 were males and 636 were females. The demographic characteristics are shown in Table 1.

In 648 (50.6\%) of the hospitalized patients with COVID-19, proteinuria was identified. Severe proteinuria was observed in 38 patients (3.0\%); mild proteinuria was identified in 610 (94.1\%). In 34 patients with COVID-19 infection, proteinuria was newly developed. Proteinuria increased in 22 patients. In contrast, proteinuria disappeared or dramatically decreased during the hospitalization in 223 patients. Hematuria was detected in 77 patients $(6.0 \%)$. Leukocyturia was detected in 282 hospitalized patients (22.0\%).

Potassium levels of $<3.5 \mathrm{mmol} / \mathrm{L}$ were observed in $113 \mathrm{pa}$ tients (8.8\%). In 90 patients with hypokalemia, mild proteinuria ( $>0.15 \mathrm{~g} / \mathrm{g} \mathrm{Cr}$ ) was detected, manifested by hematuria in 15 patients and by leukocyturia in 41 patients. In 23 patients, there were no changes in the urine tests. In 51 patients, hypokalemia was associated with AKI (32 patients, stage $1 ; 12$ patients, stage 2 ; seven patients, stage 3 ).

\section{Incidence and severity of acute kidney injuries}

The cumulative frequency of AKI was 371 of 1,280 patients (29.0\%). Ten of these patients $(2.7 \%)$ required dialysis (Supplementary Fig. 1, available online). The proportions of patients with AKI stages 1, 2, and 3 among the hospitalized patients were 256 (69.0\%), 68 (18.3\%), and 47 (12.7\%), respectively.

AKI was diagnosed in 63 of 134 patients (47.0\%) admitted to the intensive care unit (ICU). The numbers of AKI patients 
Table 1. Characteristics of the study subjects

\begin{tabular}{|c|c|c|c|c|}
\hline Variable & Total $(n=1,280)$ & Without AKI $(n=909)$ & $\mathrm{AKI}(\mathrm{n}=371)$ & $p$-value ${ }^{a}$ \\
\hline \multicolumn{5}{|l|}{ Characteristic } \\
\hline Age (yr) & $62.8(52-75)$ & $62.0(52-74)$ & $65.0(53-78)$ & 0.007 \\
\hline Male sex & $645(50.4)$ & 455 (50.1) & $190(51.2)$ & 0.70 \\
\hline Arterial hypertension & $373(29.1)$ & $179(19.7)$ & $194(52.3)$ & 0.001 \\
\hline Diabetes mellitus & $222(17.3)$ & $127(14)$ & $95(25.6)$ & 0.001 \\
\hline Obesity $^{b}$ & $495(38.7)$ & $349(38.4)$ & $146(39.3)$ & 0.75 \\
\hline BMl $\left(\mathrm{kg} / \mathrm{m}^{2}\right)$ & $29.5(24.8-32.4)$ & $29.2(24.8-32.4)$ & $30.1(24.7-32.6)$ & 0.16 \\
\hline Intensive care unit & $134(10.5)$ & $70(7.7)$ & $64(17.3)$ & 0.001 \\
\hline ARDS $^{c}$ & $31(2.4)$ & $0(0)$ & $31(8.4)$ & 0.001 \\
\hline Invasive ventilation & $26(2.0)$ & $0(0)$ & $26(7.0)$ & 0.001 \\
\hline Shock & $10(0.8)$ & $0(0)$ & $10(2.7)$ & 0.001 \\
\hline Length of hospital stay (day) & $10(8-14)$ & $10(7-13)$ & $12(8-16)$ & 0.01 \\
\hline \multicolumn{5}{|l|}{ Laboratory parameter } \\
\hline Proteinuria & $648(50.6)$ & $407(44.8)$ & $241(65.0)$ & $<0.001$ \\
\hline $\operatorname{GFR}\left(\mathrm{mL} / \mathrm{min} / 1.73 \mathrm{~m}^{2}\right)$ & 70 (45-93) & $80(58-100)$ & $41(27-47)$ & $<0.001$ \\
\hline INR $(n=1,179)$ & $1.11(1.02-1.23)$ & $1.08(0.99-1.16)$ & $1.17(1.05-1.35)$ & $<0.003$ \\
\hline aPTT $(\mathrm{sec})(\mathrm{n}=906)$ & $26.2(24.7-31.0)$ & $25.2(22.0-29.7)$ & $29.3(24.8-41.8)$ & $<0.001$ \\
\hline Ferritin $(\mu g / L)(n=1,087)$ & $420.0(266-580)$ & $357.0(178-521)$ & $533.5(360-649)$ & $<0.001$ \\
\hline $\mathrm{LDH}(\mathrm{U} / \mathrm{L})(\mathrm{n}=1,217)$ & $607(458-856)$ & $543(432.5-687.5)$ & $742(518-1,105)$ & $<0.001$ \\
\hline $\operatorname{CRP}(\mathrm{mg} / \mathrm{L})(\mathrm{n}=1,280)$ & $81.0(34-135)$ & $64.2(28.9-116.9)$ & $122.4(70.2-197.9)$ & $<0.001$ \\
\hline D-dimer (mg/L) $(n=760)$ & $0.3(0.03-0.60)$ & $0.1(0.02-0.48)$ & $0.4(0.04-0.94)$ & $<0.001$ \\
\hline
\end{tabular}

Data are presented as median (interquartile range) or number (\%).

AKI, acute kidney injury; aPTT, activated partial thromboplastin time; ARDS, acute respiratory distress syndrome; BMI, body mass index; CRP, C-reactive protein; GFR, glomerular filtration rate; INR, international normalized ratio; LDH, lactate dehydrogenase.

${ }^{a}$ Differences in proportions and continuous data were tested using the Pearson chi-square test and Mann-Whitney $U$ test, respectively. ${ }^{b}$ Obesity is defined as having a $\mathrm{BMI} \geq 30.0 \mathrm{~kg} / \mathrm{m}^{2}$. ${ }^{\mathrm{C}} \mathrm{ARDS}$ was diagnosed in cases of $\mathrm{O}_{2}$ saturation of $<92 \%$ and the need for respiratory support.

with stages 2 and 3 were higher than those in the common group of hospitalized patients (17 [27\%] and 11 [17.5\%], respectively). In 96 patients of 371 patients (25.9\%), the $\mathrm{Cr}$ levels had already increased at the time of admission $1.62 \mathrm{mg} /$ dL (IQR, 1.33-1.80 mg/dL).

According to a factorial analysis and the rotation method of varimax with Kaiser normalization, we identified three groups of AKI risk factors as follows: 1) inflammation (an augmentation of CRP and ferritin levels), 2) an increase in aPTT values, and 3) age of $>65$ years. The linear regression model showed the significance of AKI development with the following parameters; older age, glomerular filtration rate (GFR) on hospital admission, degree of CRP increase, ferritin levels, the lengthening of the aPTT in dynamics from baseline to maximum laboratory values, the maximum values of D-dimer during hospitalization and the development of ARDS (Table 2).

\section{Outcomes}

Overall, 162 of the 1,280 hospitalized patients (12.7\%) and 111 of the 371 patients (29.9\%) with AKI did not survive. In total, 860 of 907 patients (94.8\%) without AKI survived, while 258 patients in the AKI group (23.1\%) survived (Supplementary Table 1, available online). According to the Kaplan-Meier analysis, the survival probability gradually decreased among patients with AKI 1, 2, and 3 in the general group (Fig. 1A). Fifty-two patients (73.2\%) without AKI and 34 patients (39.5\%) with AKI survived in the ICU (Supplementary Table 2 , available online).

In the ICU, the survival probability was significantly lower in patients with AKI 3 than it was in those without AKI, or with AKI 1 or 2 . There was a significant difference between the AKI 3, AKI 2 and no AKI groups. However, there was no difference in the survival probability between the no AKI, AKI 1, and AKI 2 groups (Fig. 1B).

The only difference between the AKI 2 and 3 groups was 
Table 2. Linear regression model of AKI risk in COVID-19 patients

\begin{tabular}{|c|c|c|c|c|}
\hline Variable & Standardized coefficients, $\otimes$ (beta) & Unstandardized coefficients, $\otimes$ (beta) & p-value & $95 \% \mathrm{Cl}$ for $\mathrm{B}$ \\
\hline Age & 0.602 & 0.005 & 0.001 & 0.004 to 0.006 \\
\hline aPTT & 0.108 & 0.004 & 0.001 & 0.002 to 0.005 \\
\hline CRP & 0.343 & 0.002 & 0.003 & 0.001 to 0.002 \\
\hline GFR & -0.479 & -0.003 & 0.001 & -0.004 to -0.003 \\
\hline ARDS & 0.084 & 0.053 & 0.03 & 0.100 to 0.005 \\
\hline
\end{tabular}

The covariates: age, sex, body mass index, changes in international normalized ratio, aPTT, CRP, ferritin, fibrinogen, D-dimer, lactate dehydrogenase levels over time from baseline to maximum laboratory values, maximum D-dimer, maximum proteinuria, GFR on admission, computed tomography stage, ARDS, disseminated intravascular coagulation, diabetes mellitus, and arterial hypertension are entered in the linear regression model.

AKI, acute kidney injury; aPTT, activated partial thromboplastin time, ARDS, acute respiratory distress syndrome; CI, confidence interval; COVID-19, coronavirus disease 2019; CRP, C-reactive protein; GFR, glomerular filtration rate.

A

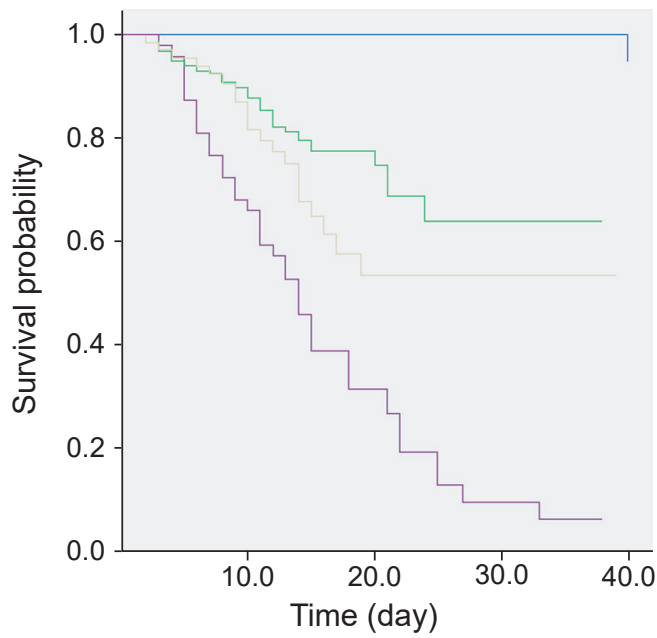

B

$\neg$ No AKI

$\neg$ AKI stage 1

AKI stage 2

$\neg$ AKI stage 3

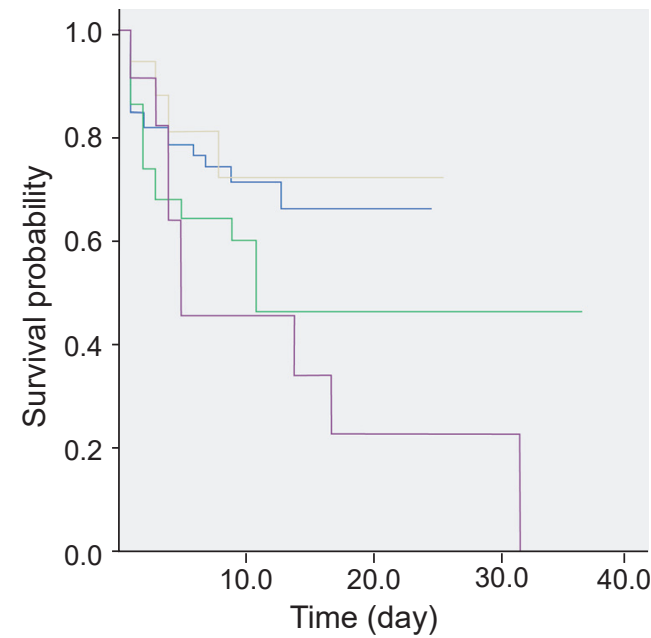

$\neg$ No AKI

$\neg$ AKI stage 1

AKI stage 2

$\neg$ AKI stage 3

Figure 1. Mortality in COVID-19 patients without AKI and with AKI stages 1, 2, and 3. (A) In the general group. No AKI vs. AKI stages 1, 2, and 3, p < 0.001; AKI stage 1 vs, AKI stage 3, p < 0.001; AKI stage 2 vs. AKI stage 3 not significant ( $p>0.05)$. (B) In the intensive care unit. No AKI vs. AKI stage 3, p < 0.001; AKI stage 2 vs. AKI stage 3, p < 0.001. By Mantel-Cox log-rank test.

AKI, acute kidney injury, COVID-19, coronavirus disease 2019.

the D-dimer level. The D-dimer level was significantly higher in patients with AKI $3(4.6[0.87-6] \mathrm{mg} / \mathrm{L})$ than it was in patients with AKI $2(0.75[0.34-1.91] \mathrm{mg} / \mathrm{L}, \mathrm{p}<0.05)$ and in patients without AKI $(0.27[0.08-1.67] \mathrm{mg} / \mathrm{L}, \mathrm{p}<0.001)$.

Cox regression univariate analysis was performed on data from 1,280 COVID-19 patients. The incidence rate of death was higher in the COVID-19 patients with AKI than it was in those without AKI. The HR for AKI patients compared with the non-AKI patients was 3.96 (95\% CI, 2.83-5.54, p < 0.0001; AKI stage 1: 1.40 [0.99-1.97], $\mathrm{p}=0.06$; AKI stage 2: 1.99 [1.24-3.17], p < 0.004; AKI stage 3: 6.01 [4.14-8.71], p < 0.0001) which indicates that the mortality of patients with AKI was higher than that of non-AKI patients.

In multivariate Cox proportional hazards analysis, the following clinical features were predictors of unfavorable outcomes in COVID-19 patients; AKI 3 stage, lower GFR on admission, DIC with elongation of aPTT, and arterial hypertension (Fig. 2).

\section{Discussion}

Renal damage in COVID-19 results from the interaction of many different pathological processes. This renal damage may be due to the direct cytotoxic effect of the virus on kid- 


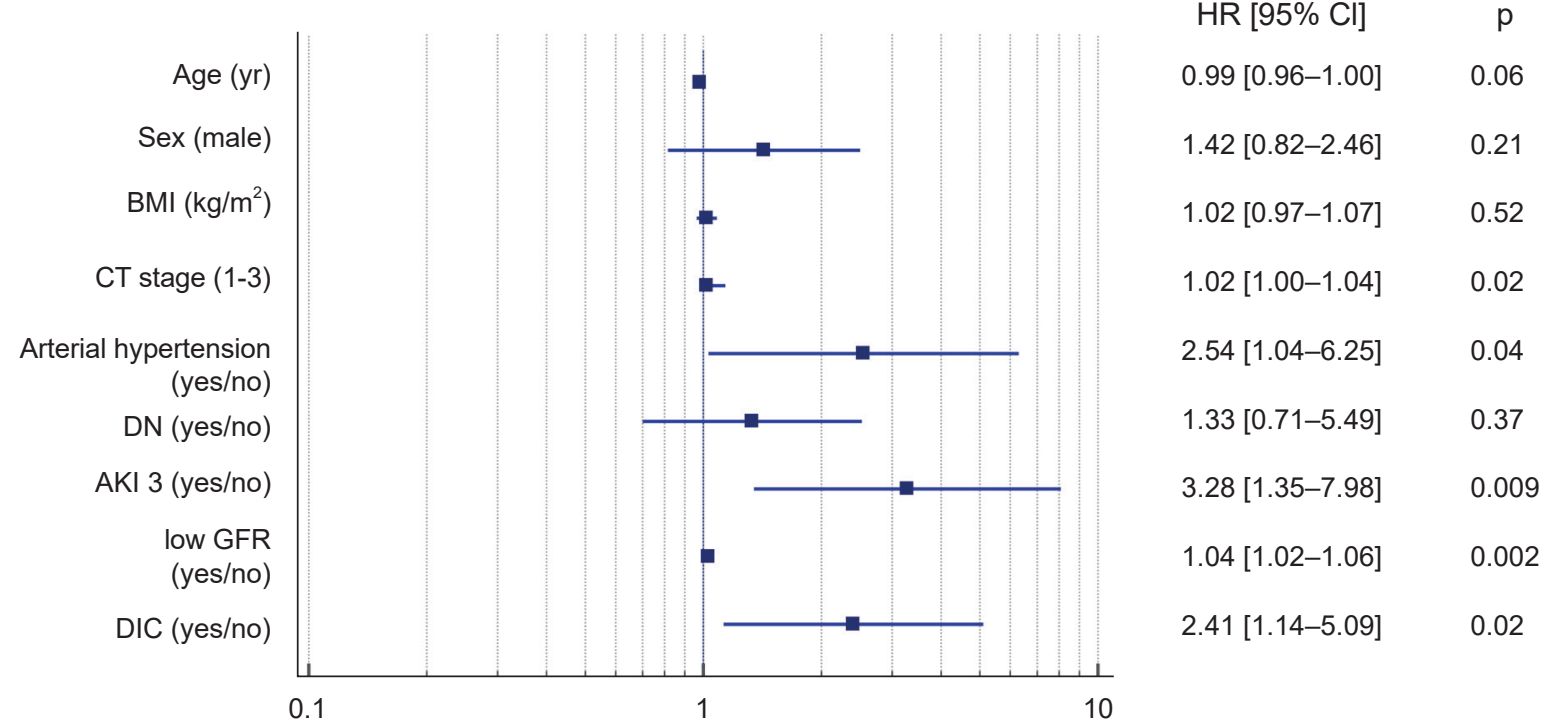

Figure 2. Multivariate Cox regression model for in-hospital mortality in COVID-19 patients. The covariates are age; sex; body mass index (BMI); glomerular filtration rate (GFR) at admission (baseline); international normalized ratio, C-reactive protein, ferritin, fibrinogen, and lactate dehydrogenase levels over time; maximum proteinuria; computed tomography stage; disseminated intravascular coagulation (DIC); ARDS; AKI, AKI stage 1, 2, and 3; diabetes mellitus (DM); and arterial hypertension were entered into the Cox regression model. Hazard ratios $(\mathrm{HR})$ with $95 \%$ confidence intervals $(\mathrm{Cl})$ for mortality were calculated. The $\mathrm{p}$-values of $<0.05$ were considered statistically significant.

AKI, acute kidney injury; COVID-19, coronavirus disease 2019.

ney cells, given that viral particles have been identified in podocytes, tubular epithelium, and endothelial cells $[10,11]$. Tubular damage may also be associated with a direct toxic effect of high cytokine concentrations, which lead to tubular dysfunction or tubular necrosis. The SARS-CoV-2 virus recruits CD68+ macrophages into the tubulointerstitium. This suggests that proinflammatory cytokines derived from macrophages induce tubular damage [11].

The effect of the virus on the podocyte explains a high percentage of the proteinuria levels in patients affected by COVID-19. However, proteinuria is often transient and rarely exceeds $3 \mathrm{~g}$ in this setting. In our study, proteinuria was detected in half of the COVID-19 patients and was a common manifestation of kidney damage.

Rare cases of collapsing nephropathy with nephrotic syndrome have been described in African American patients. A relationship between the APOLl genotype with the mutation of the APOL1 gene has been advocated [12,13]. The majority of patients in our study had transient proteinuria, which may indicate its association with COVID-19 infection. Other observed changes in the urinary sediment analysis included hematuria and leukocyturia. It should be noted that the frequency of proteinuria was comparable with other similar studies; nevertheless, we evidenced that hematuria was present less often $[3,14]$. Leukocyturia was detected more frequently than was hematuria in our results. However, it is difficult to prove that there is a direct connection between the urinary sediment and viral action.

Hypokalemia was observed in $8.8 \%$ of patients. Our data do not match the data of a prior study, which showed that hypokalemia occurs in $93 \%$ of patients at the time of admission. The reasons for hypokalemia in COVID-19 patients are discussed. Chen et al. [15] identified a connection between hypokalemia and kaliuresis, which were identified in some patients with COVID-19. Gaetano et al. [16] suggested that hypokalemia may be related to kaliuresis due to increased angiotensin II levels, and uptake of diuretics and other nephrotoxic drugs. However, there is insufficient evidence to interpret kaliuresis as a result of tubular damage caused by COVID-19 infection.

The second most common renal lesion with COVID-19 was AKI, with a cumulative incidence of $29 \%$. However, the sum of the incidences of stage 2 and stage 3 was $9 \%$. According to previous studies, the incidence of AKI varies widely 
from $0.5 \%$ to $29 \%[1,2,17,18]$.

The well-known risk factors for AKI are underlying diseases such as diabetes mellitus, systemic arterial hypertension, elderly age, and/or chronic kidney disease [19]. We noticed a direct, proportional relationship between inflammatory marker levels (CRP, ferritin, LDH) and AKI development. The independent prognostic elements of AKI were older age, increased CRP, ferritin, and aPTT lengthening. The findings suggest that in addition to the severity of inflammation in COVID-19, there are other important factors in kidney damage that activate the clotting chain. We also identified a significant increase in D-dimer levels along with aPTT extension and ARDS development in the AKI group. Previous studies found that COVID-19 can induce a hypercoagulable state that is associated with progression of respiratory failure and poor prognosis $[10,11]$. Endothelial-induced injury caused by the virus results in activated complement, which contributes to the augmentation of thrombotic microangiopathy. SARS-CoV-2 is known to activate the complement lectin pathway [19]. The complement activation reaction in patients with COVID-19 was confirmed by the detection of deposits of C5b-9 (membrane attack complex), C4d, and mannose binding lectin-associated serine protease 2 in the vessels of the skin, lungs, and kidney tissues [20].

In a previous series of autopsies of patients with COVID-19, there was pronounced infiltration of lung and kidney tissues by macrophages and neutrophils [11]. Activation of innate immunity by the virus (macrophages and neutrophils) promotes the release of tissue factors and activation of the extrinsic coagulation pathway. This activation leads to the release of the protease network by neutrophils, which is the so-called neutrophil extracellular trap process (NETosis). This process promotes the activation of the intrinsic coagulation pathway [21,22].

Drawing analogies with macrophage activation syndrome, the association of immune inflammation with local or systemic blood coagulation in COVID-19 has been defined as an immunothrombotic process. This process initially starts in the lung (pulmonary intravascular coagulation), but by wide decompensation, can progress to DIC $[23,24]$.

We identified an association between extended aPTT and high D-dimer levels without a tendency to decrease in patients with AKI. Similar results were obtained by Cheng et al. [14]. Patients with AKI were found to have coagulation abnormalities, including prolonged aPTT, higher D-dimer levels, and lower platelet counts. An additional adverse factor for developing AKI is hypoxia. The evidence that suggests that hypoxia contributes to AKI development comes from a comparison of the percentage of lung tissue involved and the decrease in oxygen saturation rates in patients with AKI [25]. In our study, ARDS was found to be an independent risk factor for AKI.

A lower GFR on admission and the presence of AKI significantly increased the risk of death in patients with COVID-19; these findings were comparable to published data $[3,5,11,14]$. However, factors other than AKI also influence the mortality rate of critically ill patients, as evidenced by the absence of differences between AKI stages 1, 2 and no AKI. In addition to the severity of the COVID-19 infection itself, the presence of arterial hypertension and DIC can also increase mortality.

Our study has several limitations. In many patients, the $\mathrm{Cr}$ levels were missed before hospital admission. Therefore, we were not able to estimate the incidence of chronic kidney disease. We also did not evaluate the levels of sodium, magnesium, chlorine, or calcium in the serum. These measurements may have allowed for more reliable conclusions about the degree of tubular disorders in our patients. We did not evaluate the degree of hypovolemia or the administration of nephrotoxic drugs in our cohort. However, because the patients were admitted to the hospital, these additional AKI factors were quickly corrected and should not have affected our results. The dynamics of proteinuria can only be traced in some patients with COVID-19 infection, which did not allow us to accurately judge the direct relationship of proteinuria with COVID-19 infection.

In conclusion, acute kidney damage is a serious complication of COVID-19, because it is a predictor of increased mortality. Two factors that influence AKI development are old age and hyperactivation of the inflammatory response. In addition, DIC and the severity of lung involvement were associated with severe AKI development. An increased risk of mortality in patients with COVID-19 was connected to the same factors and the presence of arterial hypertension.

\section{Conflicts of interest}

All authors have no conflicts of interest to declare. 


\section{Acknowledgments}

We would like to acknowledge Xavier Delgadillo MD and Tanya Oes, La Chaux-de-Fonds, Switzerland for their help with editing, transcribing, and correcting the English structure of this article.

\section{Authors' contributions}

Conceptualization: NC

Data curation: NC

Formal analysis: NC, SB

Investigation: $\mathrm{SB}, \mathrm{AB}, \mathrm{TA}, \mathrm{ML}$

Writing-original draft: NC, ML

Writing-review \& editing: SM

All authors read and approved the final manuscript.

\section{ORCID}

Natalia Chebotareva, https://orcid.org/0000-0003-2128-8560 Svetlana Berns, https://orcid.org/0000-0003-1002-1895 Angelina Berns, https://orcid.org/0000-0002-6838-0754 Tatyana Androsova, https://orcid.org/0000-0002-9951-126X Marina Lebedeva, https://orcid.org/0000-0002-5923-1837 Sergey Moiseev, https://orcid.org/0000-0002-7232-4640

\section{References}

1. Wang D, Hu B, Hu C, et al. Clinical characteristics of 138 hospitalized patients with 2019 novel coronavirus-infected pneumonia in Wuhan, China. JAMA 2020;323:1061-1069.

2. Guan WJ, Ni ZY, Hu Y, et al. Clinical characteristics of coronavirus disease 2019 in China. N Engl J Med 2020;382:1708-1720.

3. Li Z, Wu M, Yao J, et al. Caution on kidney dysfunctions of COVID-19 patients. medRxiv 2020 Mar 27 [Preprint]. DOI: 10. 1101/2020.02.08.20021212.

4. Wang L, Li X, Chen H, et al. Coronavirus disease 19 infection does not result in acute kidney injury: an analysis of 116 hospitalized patients from Wuhan, China. Am J Nephrol 2020;51:343348.

5. Yang X, Yu Y, Xu J, et al. Clinical course and outcomes of critically ill patients with SARS-CoV-2 pneumonia in Wuhan, China: a single-centered, retrospective, observational study. Lancet Respir Med 2020;8:475-481.

6. Cha RH, Joh JS, Jeong I, et al. Renal complications and their prognosis in Korean patients with Middle East Respiratory Syn-
drome-Coronavirus from the central MERS-CoV designated hospital. J Korean Med Sci 2015;30:1807-1814.

7. Huang JW, Chen KY, Tsai HB, et al. Acute renal failure in patients with severe acute respiratory syndrome. J Formos Med Assoc 2005;104:891-896.

8. Salehi S, Abedi A, Balakrishnan S, Gholamrezanezhad A. Coronavirus disease 2019 (COVID-19): a systematic review of imaging findings in 919 patients. AJR Am J Roentgenol 2020; 215:87-93.

9. Kidney Disease: Improving Global Outcomes (KDIGO) Acute Kidney Injury Work Group. KDIGO clinical practice guideline for acute kidney injury. Kidney Int Suppl 2012;2:1-138.

10. Su H, Yang M, Wan C, et al. Renal histopathological analysis of 26 postmortem findings of patients with COVID-19 in China. Kidney Int 2020;98:219-227.

11. Diao B, Wang C, Wang R, et al. Human kidney is a target for novel severe acute respiratory syndrome coronavirus 2 (SARS-CoV-2) infection. medRxiv 2020 Apr 10 [Preprint]. DOI: 10.1101/2020. 03.04.20031120.

12. Larsen CP, Bourne TD, Wilson JD, Saqqa O, Sharshir MA. Collapsing glomerulopathy in a patient with COVID-19. Kidney Int Rep 2020;5:935-939.

13. Kissling S, Rotman S, Gerber C, et al. Collapsing glomerulopathy in a COVID-19 patient. Kidney Int 2020;98:228-231.

14. Cheng Y, Luo R, Wang K, et al. Kidney disease is associated with in-hospital death of patients with COVID-19. Kidney Int 2020;97:829-838

15. Chen D, Li X, Song Q, et al. Hypokalemia and clinical implications in patients with coronavirus disease 2019 (COVID-19). medRxiv 2020 Feb 29 [Preprint]. DOI: 10.1101/2020.02.27.20028530 .

16. Gaetano A, Annachiara F, Francesco F, et al. Hypokalemia in patients with COVID-19. medRxiv 2020 Jun 18 [Preprint]. DOI: 10.1101/2020.06.14.20131169.

17. Huang C, Wang Y, Li X, et al. Clinical features of patients infected with 2019 novel coronavirus in Wuhan, China. Lancet 2020;395:497-506.

18. Mou Z, Zhang X. The dangers of acute kidney injury in COVID-19 patients: A meta-analysis of first reports. medRxiv 2020 May 14 [Preprint]. DOI: 10.1101/2020.04.29.20079038.

19. Zhou Y, Lu K, Pfefferle S, et al. A single asparagine-linked glycosylation site of the severe acute respiratory syndrome coronavirus spike glycoprotein facilitates inhibition by mannose-binding lectin through multiple mechanisms. J Virol 2010;84:8753-8764.

20. Magro C, Mulvey JJ, Berlin D, et al. Complement associated microvascular injury and thrombosis in the pathogenesis of 
severe COVID-19 infection: a report of five cases. Transl Res 2020;220:1-13.

21. Jackson SP, Darbousset R, Schoenwaelder SM. Thromboinflammation: challenges of therapeutically targeting coagulation and other host defense mechanisms. Blood 2019;133:906-918.

22. Zucoloto AZ, Jenne CN. Platelet-neutrophil interplay: insights into neutrophil extracellular trap (NET)-driven coagulation in infection. Front Cardiovasc Med 2019;6:85.

23. McGonagle D, Sharif K, O'Regan A, Bridgewood C. The role of cytokines including interleukin-6 in COVID-19 induced pneu- monia and macrophage activation syndrome-like disease. Autoimmun Rev 2020;19:102537.

24. Henry BM, Vikse J, Benoit S, Favaloro EJ, Lippi G. Hyperinflammation and derangement of renin-angiotensin-aldosterone system in COVID-19: a novel hypothesis for clinically suspected hypercoagulopathy and microvascular immunothrombosis. Clin Chim Acta 2020;507:167-173.

25. Ronco C, Reis T. Kidney involvement in COVID-19 and rationale for extracorporeal therapies. Nat Rev Nephrol 2020;16:308-310. 